\section{1-бөлім}

\section{Математика}

\section{Раздел 1}

Математика

\section{Section 1}

\section{Mathematics}

IRSTI 27.39

\title{
Arens Algebras and Matricial Spaces
}

\author{
Denis Potapov ${ }^{1}$ and Fedor Sukochev ${ }^{2}$ \\ ${ }^{1}$ School of Mathematics and Statistics, University of New South Wales, Kensington, \\ NSW 2052 Australia, E-mail:d.potapov@unsw.edu.au \\ ${ }^{2}$ School of Mathematics and Statistics, University of New South Wales, Kensington, \\ NSW 2052 Australia, E-mail: f.sukochev@unsw.edu.au
}

Let $\mathcal{M}$ be a finite von Neumann algebra equipped with a finite faithful normal trace $\tau$ and let $L^{p}(\mathcal{M}, \tau)$ be the corresponding noncommutative $L^{p}$ space of $\tau$-measurable operators associated with the couple $(\mathcal{M}, \tau), 1 \leq p<\infty$. Let $M_{N}$ be the algebra of all complex $N \times N$-matrices equipped with the standard trace $\mathrm{Tr}$. In this note we study the properties of Arens "algebras" over finite dimensional matrix spaces, given by Trunov's construction for noncommutative $L^{p}$-spaces. In this work we show that the Arens "algebras" built upon Trunov's noncommutative $L^{p}$-spaces fails to form an algebra in general. We also show that the Arens space $L^{\omega}(\alpha, h)$, with $0 \leq \alpha \leq 1$, fails to form an algebra in general, even in the setting of finite algebras associated to a trace, in contrast to $L^{\omega}(\mathcal{M}, \tau)$. In particular, we provide an example of a finite von Neumann algebra, with an associated trace, such that $L^{\omega}(\alpha, h)$ is not an algebra, for any choice of $\alpha \in[0,1]$.

Keywords: von Neumann algebra, finite trace, Arens "algebras", Noncommutative $L^{p}$-spaces.

\section{Алгебры Аренса и матричные пространства}

Денис Потапов ${ }^{1}$ и Федор Сукочев ${ }^{2}$

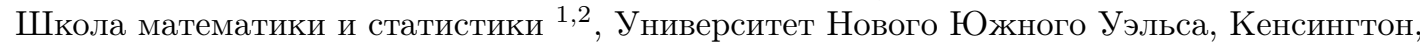
НЮУ 2052 Австралия

Пусть $\mathcal{M}$ - конечная алгебра фон Неймана, снабженная конечным точным нормальным следом $\tau$ и пусть $L^{p}(\mathcal{M}, \tau)$ - соответствующее некоммутативное пространство $L^{p} \tau$ измеримых операторов, связанных с парой $(\mathcal{M}, \tau), 1 \leq p<\infty$. Пусть $M_{N}$ - алгебра всех комплексных $N \times N$-матриц, снабженных со стандартным следом $T r$. В этой заметке мы изучаем свойства "алгебр" Аренса над конечномерными матричными постранствами, заданные конструкцией Трунова для некоммутативного $L$-пространства. В этой работе мы покажем, что "алгебра" Аренса построена на некоммутативном $L$-пространстве Трунова не образуют алгебру. Мы также показываем, что пространство Аренса $L^{\omega}(\alpha, h)$, с $0 \leq \alpha \leq 1$, не образует алгебру, даже в случае когда алгебра конечная, связанных со следом, в отличие от $L^{\omega}(\mathcal{M}, \tau)$. В частности, мы приводим пример конечной алгебры фон Неймана с связанный следом, такой, что $L^{\omega}(\alpha, h)$, не является алгеброй, для любого выбора $\alpha \in[0,1]$.

Ключевые слова: алгебра фон Неймана, конечный след, "алгебры" Аренса, некоммутативные $L^{p}$-пространства.

\section{Introduction}

Given a von Neumann algebra with a suitable trace, we may consider the now well developed theory of noncommutative integration (see, for example, $[4,9]$ ). If we do not have a trace, or wish to consider integration with respect to a state, then the theory becomes substantially more involved. We may define noncommutative $L^{p}$-spaces, however in the most common 
approaches, such as those of Haagerup [5, 10], or Connes and Hilsum [3, 6], the intersection of any two $L^{p}$-spaces for different values of $p$ is necessarily trivial.

Our goal is to better understand the properties of noncommutative Arens algebras for integration with respect to a state. Obviously these constructions with trivial intersection are not suitable, however there are alternative possibilities. In this note we study the properties of Arens "algebras" over finite dimensional matrix spaces, given by Trunov's construction for noncommutative $L^{p}$-spaces $[11,12]$. This extends our understanding of Arens' construction [2] for different noncommutative spaces. This topic has also been investigated by Albeverio, Ayupov, and Abdullaev [1], who characterise when this construction forms an algebra, for von Neumann algebras of type $\mathrm{I}_{n}$.

In this note we show that the Arens space $L^{\omega}(\alpha, h)$, with $0 \leq \alpha \leq 1$, fails to form an algebra in general, even in the setting of finite algebras associated to a trace, in contrast to $L^{\omega}(\mathcal{M}, \tau)$ [7]. In Section 2 we review the necessary background, and in Section 3 we detail some intermediate results for matrix algebras. Then in Section 4 we provide an example of a space which fails to form an algebra for $\alpha \in(0,1)$. Finally, in Section 5 we show that the example also fails to form an algebra for $\alpha \in\{0,1\}$.

\section{Preliminaries}

Let $\mathcal{M}$ be a von Neumann algebra equipped with a finite trace $\tau$ and let $L^{p}(\mathcal{M}, \tau)$ be the noncommutative $L^{p}$ space associated with the couple $(\mathcal{M}, \tau), 1 \leq p<\infty$. The norm in the space $L^{p}(\mathcal{M}, \tau)$ is given by

$$
\|x\|_{p}=\left(\tau|x|^{p}\right)^{\frac{1}{p}}
$$

Let $h \in \mathcal{M}$ be a positive operator such that $\tau(h)=1$ and let $0 \leq \alpha \leq 1$. Introduce the Trunov $L^{p}$-spaces

$$
L^{p}(\alpha, h)=\left\{x \in \tilde{\mathcal{M}}: h^{\frac{1-\alpha}{p}} x h^{\frac{\alpha}{p}} \in L^{p}(\mathcal{M}, \tau)\right\}
$$

where $\tilde{\mathcal{M}}$ is the algebra of all $\tau$-measurable operators [4]. We equip the latter space with the norm

$$
\|x\|_{p, \alpha, h}=\left\|h^{\frac{1-\alpha}{p}} x h^{\frac{\alpha}{p}}\right\|_{p}, \quad x \in L^{p}(\alpha, h) .
$$

See $[11,12]$ for further details.

It can be seen via the Hölder inequality that the space

$$
L^{\omega}(M, \tau)=\bigcap_{1 \leq p<\infty} L^{p}(\mathcal{M}, \tau) \subseteq L^{1}(\mathcal{M}, \tau)
$$

is an algebra, i.e. $x y \in L^{\omega}(\mathcal{M}, \tau)$ provided $x, y \in L^{\omega}(\mathcal{M}, \tau)$. Here we will see that the latter observation fails in general for the space

$$
L^{\omega}(\alpha, h)=\bigcap_{1 \leq p<\infty} L^{p}(\alpha, h) \subseteq L^{1}(\mathcal{M}, \tau) .
$$

In other words, the spaces $L^{\omega}(\alpha, h)$ is not an algebra in general. 


\section{Matricial case}

Let $M_{N}$ be the algebra of all complex $N \times N$-matrices equipped with the standard trace $T r$. Let us define the matrices $x_{N}$ and $h_{N}$ by setting

$$
x_{N}=\sum_{k=1}^{N} e_{k 1} \text { and } h_{N}=\sum_{k=1}^{N} 2^{-k} e_{k k},
$$

where $\left\{e_{j k}\right\} \subseteq M_{N}$ are the matrix units. If $\|\cdot\|_{p}$ stands for the $p$-th Schatten-von Neumann norm on the algebra $M_{N}$ (see, for example, [8]), then

$$
\begin{aligned}
\left\|h_{N}^{\frac{1-\alpha}{p}} x_{N} h_{N}^{\frac{\alpha}{p}}\right\|_{p} & \leq\left\|\sum_{k=1}^{N} 2^{-\frac{2}{p}(1-\alpha) k} e_{k 1}\right\|_{2} \leq \frac{1}{2}\left(\sum_{k=1}^{N} 2^{-\frac{2}{p}(1-\alpha) k}\right)^{\frac{1}{2}} \\
& \leq c_{\alpha, p}=\frac{1}{2\left(2^{\frac{2}{p}(1-\alpha)}-1\right)^{\frac{1}{2}}}
\end{aligned}
$$

The constant $c_{\alpha, p}$ is finite for every $1 \leq p<\infty$ and every $0 \leq \alpha<1$. Furthermore, the latter constant does not depend on $N$. On the other hand, we also have that

$$
\left\|h_{N}^{\frac{(1-\alpha)}{p}} x_{N}^{*} x_{N} h_{N}^{\frac{\alpha}{p}}\right\|_{p} \geq \frac{N}{2},
$$

which follows easily from calculation of $\left(h_{N}^{\frac{(1-\alpha)}{p}} x_{N}^{*} x_{N} h_{N}^{\frac{\alpha}{p}}\right)_{1,1}=\frac{N}{2}$.

\section{The example}

Let us consider the algebra $\mathcal{M}$ given by the following direct sum

$$
\mathcal{M}=\bigoplus_{N=1}^{\infty} M_{N}
$$

We equip the latter algebra with the finite trace $\tau$ defined by

$$
\tau=\sum_{N=1}^{\infty} \frac{\lambda_{N}}{N} \operatorname{Tr}, \quad \lambda=\left(\lambda_{N}\right) \in \ell_{+}^{1},
$$

where $\ell_{+}^{1}$ is the space of non-negative real valued sequences which are 1 -summable. We define the elements $x, h \in \mathcal{M}$ by setting

$$
x=\bigoplus_{N=1}^{\infty} x_{N} \text { and } h=\bigoplus_{N=1}^{\infty} h_{N} .
$$

Proposition 4.1. For $0<\alpha<1$, the space $L^{\omega}(\alpha, h)$ fails to form an algebra. 
Proof. It follows from (1) that $x, x^{*} \in L^{\omega}(\alpha, h)$ for every $0<\alpha<1$. Indeed,

$$
\|x\|_{p, \alpha, h} \leq\left(\sum_{N=1}^{\infty} \frac{\lambda_{N}}{N} c_{\alpha, p}^{p}\right)^{\frac{1}{p}}<+\infty
$$

and

$$
\left\|x^{*}\right\|_{p, \alpha, h} \leq\left(\sum_{N=1}^{\infty} \frac{\lambda_{N}}{N} c_{1-\alpha, p}^{p}\right)^{\frac{1}{p}}<+\infty .
$$

On the other hand, from (2) we also have that

$$
\left\|x^{*} x\right\|_{p, \alpha, h} \geq \frac{1}{2}\left(\sum_{N=1}^{\infty} \lambda_{N} N^{p-1}\right)^{\frac{1}{p}} .
$$

The latter quantity is infinite, subject to appropriate choice of $\lambda \in \ell^{1}$. Thus, we see that the space $L^{\omega}(\alpha, h)$ is not an algebra, if $0<\alpha<1$.

5 The case $\alpha=0$ and $\alpha=1$

Proposition 5.1. For $\alpha \in\{0,1\}$, the space $L^{\omega}(\alpha, h)$ fails to form an algebra.

Proof. Let us return to the matricial setting of Section 3. According to (1) we clearly have that

$$
\left\|h_{N}^{\frac{1}{p}} x_{N}\right\|_{p} \leq c_{p}
$$

and the constant $c_{p}$ is finite for every $1 \leq p<\infty$. Moreover, the constant $c_{p}$ does not depend on $N$.

Observe that the operators $x_{N}$ and $x_{N}^{*}$ are rank one operators and hence they are equivalent, i.e. there is a unitary operator $v_{N} \in M_{N}$ such that

$$
x_{N}^{*}=v_{N} x_{N} v_{N}^{*} \text {. }
$$

Consequently, we obtain that

$$
\left\|h_{N}^{\frac{1}{p}} v_{N} x_{N}\right\|_{p}=\left\|h_{N}^{\frac{1}{p}} x_{N}^{*}\right\|_{p} \geq \frac{1}{2} N^{\frac{1}{2}}
$$

by unitary invariance.

Indeed, this inequality follows as

$$
\left\|h_{N}^{\frac{1}{p}} x_{N}^{*}\right\|_{p}=\left\|\left(\sum_{k=1}^{N} 2^{-k} e_{k k}\right)\left(\sum_{k=1}^{N} e_{1 k}\right)\right\|_{p} \geq\left\|\sum_{k=1}^{N} \frac{1}{2} e_{1 k}\right\|_{p}=\frac{1}{2} N^{\frac{1}{2}} .
$$

Repeating the construction of Section 4 with

$$
x=\bigoplus_{N=1}^{\infty} x_{N} \text { and } v=\bigoplus_{N=1}^{\infty} v_{N}
$$

shows that $L^{\omega}(\alpha, h)$ fails to be an algebra also for $\alpha=0$. Considering $x^{*}$ and $v^{*}$ proves that this also fails for $\alpha=1$. 


\section{References}

[1] Albeverio S., Ayupov Sh. A., and Abdullaev R. Z. "Arens spaces associated with von Neumann algebras and normal states, "Positivity. 14 (2010): no.1, 105-121.

[2] Arens R. "The space $L_{w}$ and convex topological rings, "Bull. Amer. Math. Soc. 52 (1946), $931-935$.

[3] Connes A. "On the spatial theory of von Neumann algebras,"J. Funct. Anal. 35 (1980): no.2, 153-164.

[4] Fack T. and Kosaki H. "Generalized s-numbers of $\tau$-measurable operators, "Pacific J. Math. 123 (1986): no.2, 269-300.

[5] Haagerup U. " $L_{p}$-spaces associated with an arbitrary von Neumann algebra, "Proc. Colloq. Internat. CNRS. 274 (1979), 175-184.

[6] Hilsum M. "Les espaces $L_{p}$ d'une algebre de von Neumann definies par la deriv ee spatiale,"J. Funct. Anal. 40 (1981): no.2, 151-169.

[7] Kunze W. "Noncommutative Orlicz spaces and generalized Arens algebras,"Math. Nachr. 147 (1990), $123-138$.

[8] S. Lord, F. Sukochev, and D. Zanin: Singular traces. Theory and applications (De Gruyter, Berlin, 2013).

[9] M. Takesaki: Theory of operator algebras. II. (Springer-Verlag, Berlin, 2003)

[10] M. Terp: $L_{p}$ spaces associated with von neumann algebras (Notes, Math. Institute, Copenhagen Univ., 1981).

[11] Trunov N. V. "Integration in von Neumann algebras and regular weights,"In Constructive theory of functions and functional analysis. Kazan. Gos. Univ., Kazan. (1981): no.3, 73-87.

[12] Trunov N. V. " $L_{p}$ spaces associated with a weight on a semifinite von Neumann algebra, "In Constructive theory of functions and functional analysis. Kazan. Gos. Univ., Kazan. (1981): no.3, 88-93. 